\title{
ARTICLE \\ Metabolic perturbations of post-load hyperglycemia vs. fasting hyperglycemia
}

\author{
Jing-yi Lu' ${ }^{1}$, Jia-hui Peng ${ }^{1}$, Xiao-jing Ma', Yi-nan Zhang ${ }^{2}$, Wei Zhu' ${ }^{1}$, Xing-xing $\mathrm{He}^{1}$, Ling-wen Ying ${ }^{1}$, Yu-qian Bao ${ }^{1}$, Jian Zhou ${ }^{1}$ and \\ Wei-ping Jia ${ }^{1}$
}

There is evidence that post-load/post-meal hyperglycemia is a stronger risk factor for cardiovascular disease than fasting hyperglycemia. The underlying mechanism remains to be elucidated. The current study aimed to compare the metabolic profiles of post-load hyperglycemia and fasting hyperglycemia. All subjects received an oral glucose tolerance test (OGTT) and were stratified into fasting hyperglycemia (FH) or post-load hyperglycemia (PH). Forty-six ( $\mathrm{FH}, n=23 ; \mathrm{PH}, n=23)$ and 40 patients ( $\mathrm{FH}, n=20 ; \mathrm{PH}$, $n=20$ ) were recruited as the exploratory and the validation set, respectively, and underwent metabolic profiling. Eighty-seven subjects including normal controls (NC: $n=36$; FH: $n=22 ; \mathrm{PH}: n=29$ ) were additionally enrolled and assayed with enzyme-linked immunosorbent assay (ELISA). In the exploratory set, 10 metabolites were selected as differential metabolites of PH (vs. FH). Of them, mannose and 5-aminoimidazole-4-carboxamide ribonucleotide (AICAR) were confirmed in the validation set to be significantly higher in FH than in PH. In the 87 subjects measured with ELISA, FH had numerically higher mannose $(466.0 \pm 179.3$ vs. $390.1 \pm 140.2 \mathrm{pg} / \mathrm{ml})$ and AICAR $(523.5 \pm 164.8 \mathrm{vs} .512 .1 \pm 186.0 \mathrm{pg} / \mathrm{ml})$ than did PH. In the pooled dataset comprising 173 subjects, mannose was independently associated with FPG $(\beta=0.151, P=0.035)$ and HOMA-IR $(\beta=0.160, P=0.026)$, respectively. The associations of AICAR with biochemical parameters did not reach statistical significance. $\mathrm{FH}$ and PH exhibited distinct metabolic profiles. The perturbation of mannose may be involved in the pathophysiologic disturbances in diabetes.

Keywords: Type 2 diabetes; insulin resistance; insulin secretion; metabolomics

Acta Pharmacologica Sinica (2019) 40:216-221; https://doi.org/10.1038/s41401-018-0018-6

\section{INTRODUCTION}

Diabetes is associated with a high incidence of cardiovascular disease (CVD) [1], and poor control of hyperglycemia plays a significant role in the development of CVD in diabetes patients [2]. Accumulating evidence indicates that fasting and post-meal/postload hyperglycemia $(\mathrm{PH})$ do not contribute equally to the development of CVD, with the latter being a stronger risk factor. Given the tight link between post-meal/post-load glucose and diabetes complications including CVD, the International Diabetes Federation (IDF) has specifically published a guideline for management of post-meal glucose [3]. In the Diabetes Intervention Study, which was a prospective population-based trial, Hanefeld et al. [4] reported that post-meal but not fasting blood glucose was significantly associated with myocardial infarction. Cavalot et al. [5] followed 505 diabetes patients for 14 years and observed that blood glucose $2 \mathrm{~h}$ after lunch, but not fasting blood glucose, was a significant predictor of cardiovascular events. Moreover, Coutinho et al. [6] performed a meta-analysis of published data for 95,783 subjects, and found that the glucose level at $2 \mathrm{~h}$, post-oral glucose load was a more powerful predictor of cardiovascular events than fasting glucose. Despite the different associations of fasting and post-meal/PH with CVD, the mechanism underlying this phenomenon remains incompletely understood.

Metabolomics is a rapidly evolving technology by which an entire spectrum of endogenous metabolites in cells, biofluids, or tissues is measured quantitatively following genetic or environmental interventions [7]. Research indicates that metabolomics can provide important insight for biomarker discovery, toxicity evaluation, and understanding the pathogenic nature of various diseases [8-10]. For example, in 2009, Newgard et al. [8] performed metabolomic profiling of obese vs. lean humans and identified a branched-chain amino acid (BCAA)-related metabolic signature that correlated with insulin resistance. Since then, BCAA has garnered much research interest in the pathogenesis of type 2 diabetes and the BCAA-insulin resistance relationship has now been well established.

In the current study, we conducted a metabolomic analysis of serum samples from patients with fasting or $\mathrm{PH}$ in order to identify differences in the metabolic features of the two groups and achieve a better understanding of fasting and post-meal/PH.

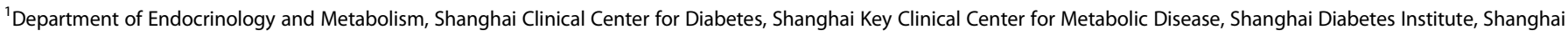

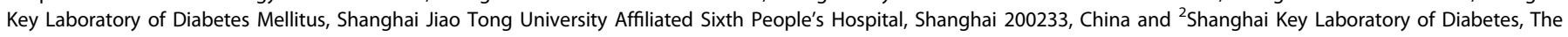
Metabolic Diseases Biobank, Center for Translational Medicine, Shanghai Jiao Tong University Affiliated Sixth People's Hospital, Shanghai 200233, China

Correspondence: Xiao-jing Ma (maxiaojing@sjtu.edu.cn) or Jian Zhou (zhoujian@sjtu.edu.cn)

These authors contributed equally: Jing-yi Lu, Jia-hui Peng.
}

Received: 6 September 2017 Accepted: 11 February 2018

Published online: 17 May 2018 


\section{MATERIALS AND METHODS}

Study population

Participants without a prior history of diabetes were enrolled consecutively from the outpatient clinic of the Department of Endocrinology and Metabolism of Shanghai Jiao Tong University Affiliated to the Sixth People's Hospital. Each participant received a $75-g$ oral glucose tolerance test (OGTT). Subjects with both fasting plasma glucose (FPG) $\geq 7.0 \mathrm{mmol} / \mathrm{L}$ and 2 - $\mathrm{h}$ plasma glucose $(2-h$ PG) $\geq 11.1 \mathrm{mmol} / \mathrm{L}$ were excluded from the study. The remaining individuals were further stratified into two groups: a fasting hyperglycemia (FH) group and a $\mathrm{PH}$ group. $\mathrm{FH}$ was defined as $F P G \geq 7.0 \mathrm{mmol} / \mathrm{L}$ but $2-\mathrm{h} P G<11.1 \mathrm{mmol} / \mathrm{L}$, whereas $\mathrm{PH}$ was defined as FPG $<7.0 \mathrm{mmol} / \mathrm{L}$ but $2-\mathrm{h} P G \geq 11.1 \mathrm{mmol} / \mathrm{L}$. The two groups were further matched by age, gender, body mass index (BMI), and glycated hemoglobin $\left(\mathrm{HbA}_{1 \mathrm{c}}\right)$. Finally, 46 patients $(\mathrm{FH}$ group, $n=23$; $\mathrm{PH}$ group, $n=23$ ) were included in the exploratory set for metabolic profiling.

Next, 40 individuals (FH group, $n=20$; PH group, $n=20$ ) were enrolled as a validation set following the same inclusion, exclusion, and matching criteria to validate the results obtained from the exploratory set. Moreover, another 87 subjects were recruited and were assayed with enzyme-linked immunosorbent assay (ELISA). These included 22 with $\mathrm{FH}, 29$ with $\mathrm{PH}$, and 36 normal controls (NCs), which were defined as FPG $<5.6 \mathrm{mmol} / \mathrm{L}$ and 2-h PG $<7.8 \mathrm{mmol} / \mathrm{L}$ during OGTT. In total, 173 subjects were included in the study.

The Institutional Review Board of Shanghai Jiao Tong University Affiliated to the Sixth People's Hospital approved this study. The study was conducted in accordance with the principles of the Declaration of Helsinki II. Each participant provided written informed consent.

Anthropometric and biochemical measurements

After a fasting venous blood sample was collected, each participant received a 75-g OGTT. Plasma glucose levels were measured using the glucose oxidase method. BMI was calculated as weight divided by height squared $\left(\mathrm{kg} / \mathrm{m}^{2}\right) . \mathrm{HbA}_{1 \mathrm{c}}$ was detected by high-performance liquid chromatography (Variant II hemoglobin analyzer; Bio-Rad, Hercules, CA, USA). Electrochemiluminescence immunoassay was used to quantify serum insulin levels on a Cobas e 601 analyzer (Roche Diagnostics GmbH, Mannheim, Germany).

The homeostasis model assessments for IR index (HOMA-IR) and beta cell function (HOMA-B) were calculated using the following formulae: HOMA-IR = fasting insulin (FINS) (in $\mathrm{mU} / \mathrm{L}$ ) $\times \mathrm{FPG}$ (in $\mathrm{mmol} / \mathrm{L}$ ) $/ 22.5$; and HOMA-B $=20 \times \mathrm{FINS}$ (in $\mathrm{mU} / \mathrm{L}$ )/(FPG (in mmol/ $\mathrm{L})$ - 3.5). Early-phase insulin secretion during OGTT was assessed by $\triangle I_{30} / \triangle G_{30}$ and calculated as: (0.5-h INS (in mU/L) - FINS)/(0.5h $P G$ (in $\mathrm{mmol} / \mathrm{L}$ ) - FPG). The insulin sensitivity index was estimated with the Cederholm equation [11] shown below, where $I_{\text {mean }}$ and $G_{\text {mean }}$ represent the mean insulin and glucose concentrations, respectively, during the OGTT.

$$
\begin{aligned}
& \text { Cederholm index }= \\
& \frac{75,000+\left(\text { Glucose }_{0}-\text { Glucose }_{120}\right) \times 180 \times 0.19 \times \text { bodymass }}{120 \times G_{\text {mean }} \times \log \left(I_{\text {mean }}\right)},
\end{aligned}
$$

Commercial ELISA kits were used for measuring mannose (TSZ, HU8747, MA, USA) and 5-aminoimidazole-4-carboxamide ribonucleotide (AICAR) (ImmunoClone, IC-AICAR-Hu, NY, USA). The assays were performed according to manufacturers' instructions. The samples were then read at an absorbance of $450 \mathrm{~nm}$ and compared against a standard curve of known concentrations.

\section{Sample preparation and metabolomic analysis}

A $100 \mu \mathrm{L}$ aliquot of serum sample was extracted with $350 \mu \mathrm{L}$ of methanol and vortexed for $10 \mathrm{~s}$. The samples were then centrifuged at 13,000 r.p.m. $\left(4^{\circ} \mathrm{C}\right)$ for $10 \mathrm{~min}$. An aliquot of the
$300 \mu \mathrm{L}$ supernatant was transferred to a glass sampling vial, and was spiked with one internal standards $(20 \mu \mathrm{L} \mathrm{L-2-chlorophenyla-}$ lanine in water, $1 \mathrm{mg} / \mathrm{ml}$ ) to vacuum dry at room temperature. The residue was derivatized using a two-step procedure. First, $60 \mu \mathrm{L}$ methoxyamine $(20 \mathrm{mg} / \mathrm{ml}$ in pyridine,) was added to the vial and kept at $80^{\circ} \mathrm{C}$ for $30 \mathrm{~min}$, second, $80 \mu \mathrm{L} \mathrm{N}, \mathrm{O}-\mathrm{Bis}$ (trimethylsilyl) trifluoroacetamide (BSTFA) (1\% trimethylchlorosilane (TMCS)) was added and kept at $70^{\circ} \mathrm{C}$ for $120 \mathrm{~min}$.

Quality control $(\mathrm{QC})$ samples were prepared by mixing equal amounts of serum from all subject samples and were run after each five-test serum samples.

Gas chromatography time-of-flight mass spectrometry (GC-TOFMS) analysis was performed with an Agilent 7890 gas chromatograph system linked to a Pegasus HT time-of-flight mass spectrometer (LECO, St. Joseph, MI, USA). The system employed a DB-5 column with $5 \%$ diphenyl and $95 \%$ dimethyl polysiloxane (J\&W Scientifc, Folsom, CA, USA). A $1 \mu \mathrm{L}$ aliquot of the analyte was injected in splitless mode. Helium was used as the carrier gas, the front inlet purge flow was $3 \mathrm{ml} / \mathrm{ml}$, and the gas flow rate through the column was $1 \mathrm{ml} / \mathrm{ml}$. The initial temperature was kept at $50^{\circ} \mathrm{C}$ for $1 \mathrm{~min}$, then raised to $310^{\circ} \mathrm{C}$ at a rate of $20^{\circ} \mathrm{C}$ per min, then kept for $6 \mathrm{~min}$ at $310^{\circ} \mathrm{C}$. The injection, transfer line and ion source temperature were 280,270 , and $220^{\circ} \mathrm{C}$, respectively. The energy was $-70 \mathrm{eV}$ in electron impact mode. The mass spectrometry data was acquired in full-scan mode with the $\mathrm{m} / \mathrm{z}$ of $50-500$ at a rate of 20 spectra per s after a solvent delay of $366 \mathrm{~s}$. The dynamic range of each metabolite identified in the study is shown in Supplemental Table 1.

Data analysis

Chroma TOF $4.3 \times$ software from the LECO Corporation and the LECO-Fiehn Rtx5 database were used for raw peak extraction, baseline filtering, calibration of the baseline, peak alignment, deconvolution analysis, peak identification, and integration of the peak area as described previously [12]. To combine the data from the two batches (i.e., the exploratory set and the validation set), the original values divided by the mean of QC samples in each batch was calculated for each individual. The orthogonal partial least squares-discriminant analysis (OPLS-DA) was performed using the SIMCA-P 13.0 software package (Umetrics, Umea, Sweden) to differentiate the $\mathrm{FH}$ group from the $\mathrm{PH}$ group. On the basis of variable importance in the projection (VIP) threshold of 1 from the OPLS-DA model, a number of metabolites responsible for the differences in the metabolic profiles of $\mathrm{FH}$ and $\mathrm{PH}$ could be obtained. In parallel, the metabolites identified by the OPLS-DA model were confirmed at a univariate level using the Wilcoxon-Mann-Whitney test $(P<0.05)$. The fold change in metabolite concentrations was obtained by comparing the mean peak intensities between two groups ( $\mathrm{PH}$ vs. $\mathrm{FH}$ ). To pool the data from both metabolomics and ELISA measurements, the values obtained by each method were transformed to unit SD: (individual value - mean)/SD. The correlations of certain metabolites with indices of insulin sensitivity/resistance and insulin secretion were evaluated with Spearman's correlation coefficient. Differences between the three groups ( $\mathrm{NC}, \mathrm{FH}$, and $\mathrm{PH}$ ) were assessed by analysis of variance for normally distributed continuous variables, and by Kruskal-Wallis test for non-normally distributed continuous variables. To adjust for confounding factors, multiple regression analysis was performed. All univariate analyses were conducted using SPSS Statistics 13 (SPSS, Chicago, IL, USA). A $P$ value $<0.05$ was considered statistically significant.

\section{RESULTS}

The baseline characteristics of patients in the exploratory set are shown in Table 1. Age, gender, $\mathrm{BMI}, \mathrm{HbA}_{1 \mathrm{c}}$, and FINS were comparable between the $\mathrm{FH}$ and $\mathrm{PH}$ groups (all $P>0.05$ ). The $\mathrm{PH}$ group had a lower FPG $(P<0.001)$ and Cederholm index $(P<$ 
$0.001)$ and a higher 2-h PG $(P<0.001)$, 2-h INS $(P=0.002)$ and HOMA-B $(P<0.001)$ than did the FH group.

Among a total of 292 variables obtained from the GC-TOF-MS spectra of serum samples, 138 metabolites were identified. Discrimination of the $\mathrm{FH}$ group from the $\mathrm{PH}$ group according to the OPLS-DA model $\left(R^{2}(X)=0.644, R^{2}(Y)=0.950, Q^{2}=0.746\right)$ using one predictive component and four orthogonal components is illustrated in Fig. 1, and a clear separation between the two groups could be observed. Metabolites with VIP $>1$ (obtained from OPLS-DA analysis) and $P<0.05$ (obtained from Wilcoxon-Mann-Whitney test) were selected as candidate markers for the discrimination between $\mathrm{FH}$ and $\mathrm{PH}$. These candidate markers included 2-deoxytetronic acid, glucoheptonic acid, mannose, tagatose, sophorose, phenyl beta-D-glucopyranoside,

Table 1. The characteristics of subjects in the exploratory set

\begin{tabular}{|c|c|c|c|}
\hline Variable & $\mathrm{FH}$ & $\mathrm{PH}$ & $P$ \\
\hline$n$ & 23 & 23 & I \\
\hline Age (years) & $52.8 \pm 7.8$ & $53.8 \pm 8.6$ & 0.500 \\
\hline Gender (male/female) & $13 / 10$ & $13 / 10$ & 1.000 \\
\hline BMI $\left(\mathrm{kg} / \mathrm{m}^{2}\right)$ & $23.5 \pm 2.3$ & $23.8 \pm 2.1$ & 0.506 \\
\hline FPG (mmol/L) & $7.6 \pm 0.5$ & $6.2 \pm 0.6$ & $<0.001$ \\
\hline 2-h PG (mmol/L) & $8.9 \pm 1.6$ & $13.4 \pm 1.8$ & $<0.001$ \\
\hline $\mathrm{HbA}_{1 \mathrm{c}}(\%)$ & $6.3 \pm 0.5$ & $6.3 \pm 0.6$ & 0.880 \\
\hline FINS (mU/L) & $9.4 \pm 4.3$ & $9.6 \pm 4.2$ & 0.766 \\
\hline 2-h INS (mU/L) & $63.7 \pm 42.6$ & $97.0 \pm 51.2$ & $<0.001$ \\
\hline HOMA-IR & $0.6 \pm 0.3$ & $0.5 \pm 0.2$ & 0.069 \\
\hline Cederholm index & $36.2 \pm 6.7$ & $26.0 \pm 4.7$ & $<0.001$ \\
\hline HOMA-B & $46.2 \pm 22.9$ & $75.5 \pm 36.2$ & $<0.001$ \\
\hline$\triangle I_{30} / \triangle G_{30}$ & $9.1 \pm 12.9$ & $6.7 \pm 5.2$ & 0.260 \\
\hline
\end{tabular}

$F H$ fasting hyperglycemia, $P H$ post-load hyperglycemia, $B M I$ body mass index, FPG fasting plasma glucose, 2-h $P G$ 2-h plasma glucose, $H b A_{1 c}$ glycated hemoglobin, FINS fasting insulin, 2-h INS 2-h insulin, HOMA-IR the homeostasis model assessment for insulin resistance, HOMA-B the homeostasis model assessment for beta cell function uridine, 3,5-dihydroxyphenylglycine, palmitic acid, and 5aminoimidazole-4-carboxamide ribonucleotide (AICAR; Table 2).

Next, we performed the metabolic analysis in independent samples (validation set: FH group, $n=20 ; \mathrm{PH}$ group, $n=20$ ) (Supplemental Table 2) for validation. Of the 10 differential metabolites mentioned above, eight metabolites did not differ significantly between patients in the $\mathrm{FH}$ and $\mathrm{PH}$ groups, whereas the levels of two metabolites (mannose and AICAR) differed significantly between the $\mathrm{PH}$ and $\mathrm{FH}$ groups (Table 2). After combining the two subsets of subjects (FH group, $n=43$; $\mathrm{PH}$ group, $n=43)$, the relative intensities for mannose $(P=0.009)$ and AICAR $(P<0.001)$ were significantly lower for the PH group than for the FH group (Fig. 2). Correlation analysis revealed that mannose was significantly associated with HOMA-B $(r=-0.262$, $P=0.022)$, whereas AICAR exhibited a positive correlation with the Cederholm index $(r=0.352, P=0.001$; Fig. 3). Upon multiple

Table 2. Differential metabolites between $\mathrm{FH}$ and $\mathrm{PH}$ in exploratory and validation sets

\begin{tabular}{|c|c|c|c|c|c|}
\hline \multirow[t]{2}{*}{ Metabolites } & \multicolumn{3}{|c|}{$\begin{array}{l}\text { Exploratory set }(n= \\
\text { 46) }\end{array}$} & \multicolumn{2}{|c|}{$\begin{array}{l}\text { Validation } \\
\text { set }(n=40)\end{array}$} \\
\hline & VIP & $\mathrm{FC}$ & $P$ & $\mathrm{FC}$ & $P$ \\
\hline 2-Deoxytetronic acid & 2.82 & 0.35 & $<0.001$ & 1.02 & 0.829 \\
\hline Sophorose & 2.45 & 0.51 & 0.001 & 1.03 & 0.626 \\
\hline Tagatose & 2.39 & 0.46 & $<0.001$ & 1.06 & 0.465 \\
\hline Glucoheptonic acid & 2.34 & 0.48 & 0.001 & 0.89 & 0.822 \\
\hline Mannose & 2.06 & 0.83 & 0.006 & 0.94 & 0.040 \\
\hline AICAR & 2.05 & 0.86 & 0.022 & 0.68 & 0.003 \\
\hline Phenyl beta-D-glucopyranoside & 1.90 & 0.50 & 0.001 & 0.98 & 0.822 \\
\hline Uridine & 1.74 & 0.55 & 0.016 & 0.65 & 0.843 \\
\hline 3,5-Dihydroxyphenylglycine & 1.33 & 0.62 & 0.040 & 1.12 & 0.705 \\
\hline Palmitic acid & 1.14 & 0.80 & 0.047 & 1.02 & 0.850 \\
\hline
\end{tabular}

AICAR 5-aminoimidazole-4-carboxamide ribonucleotide, VIP variable importance projection value, $F C$ fold change (the peak intensity of metabolite in $\mathrm{PH}$ divided by that in $\mathrm{FH}$ )

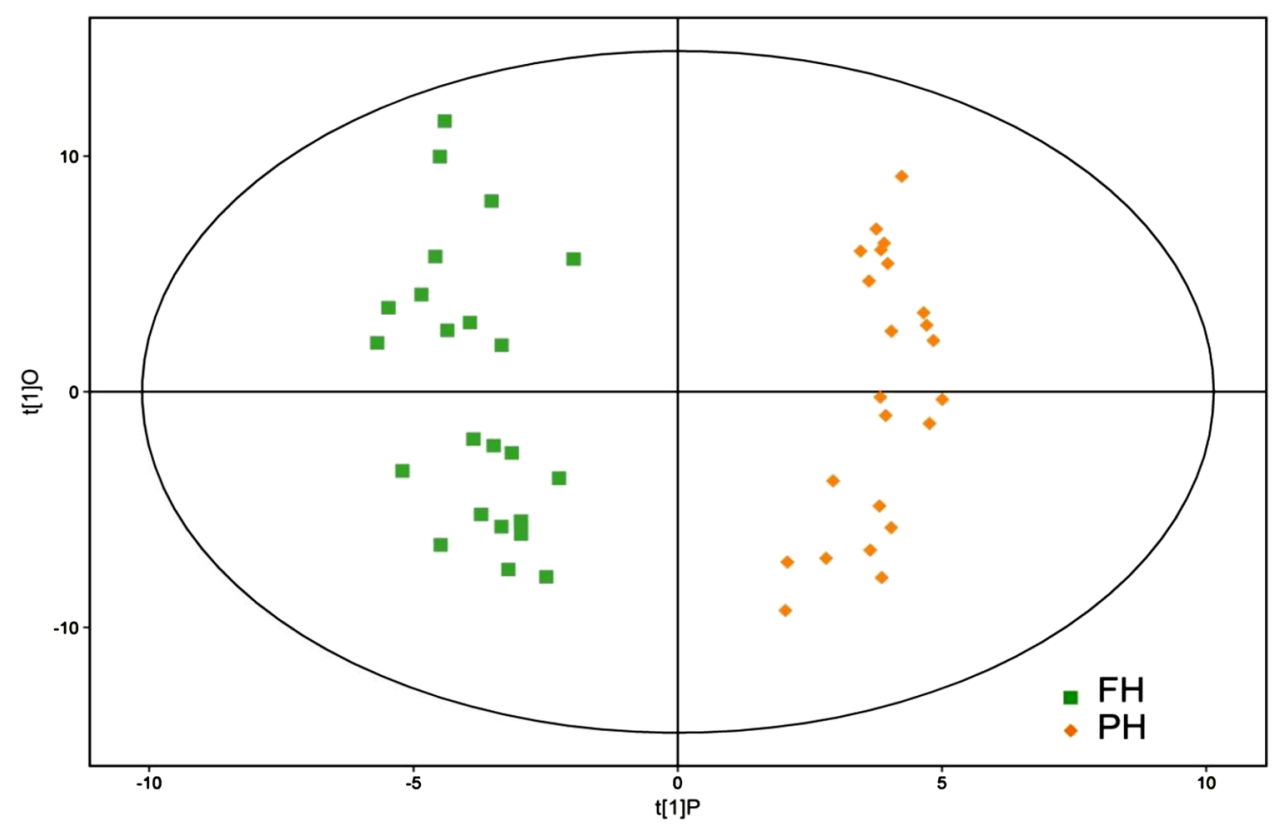

Fig. 1 Scores plot for discriminating PH from FH in the OPLS-DA model. Data is from the exploratory set 
a

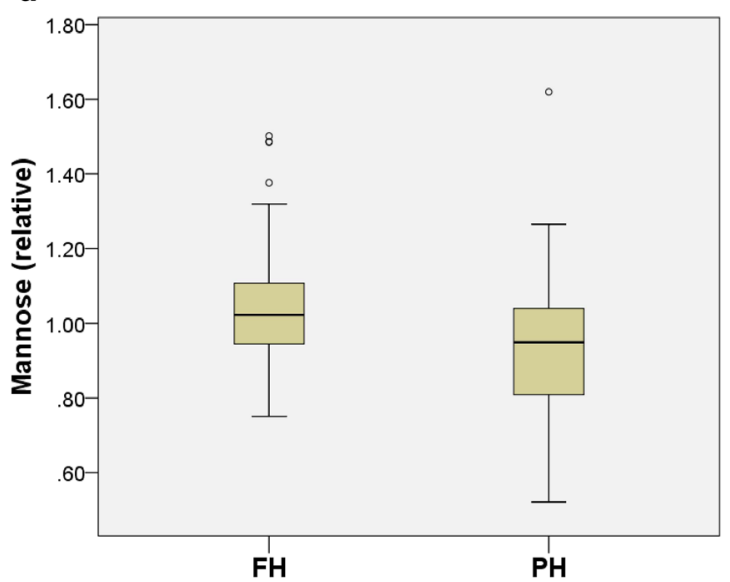

b

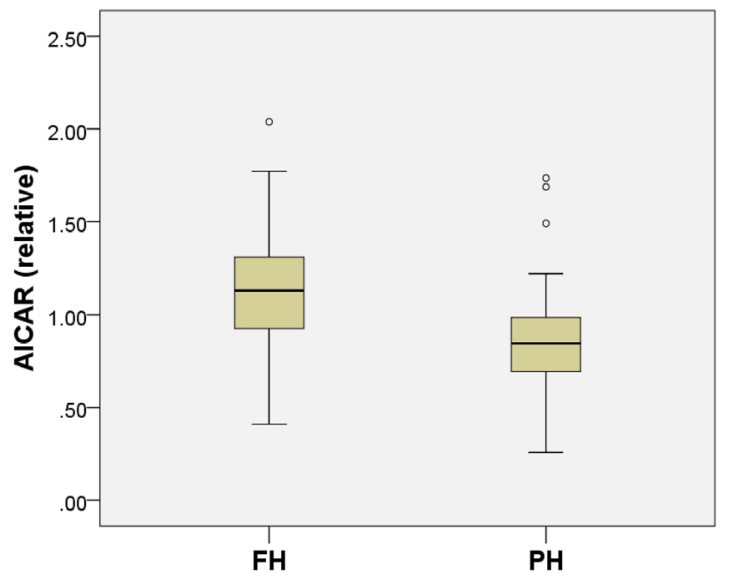

Fig. 2 Relative concentrations of mannose and AICAR in the combined set of exploratory and validation sets. (a) The distribution of mannose between $\mathrm{FH}$ and $\mathrm{PH}$; (b) the distribution of AICAR between $\mathrm{FH}$ and $\mathrm{PH}$
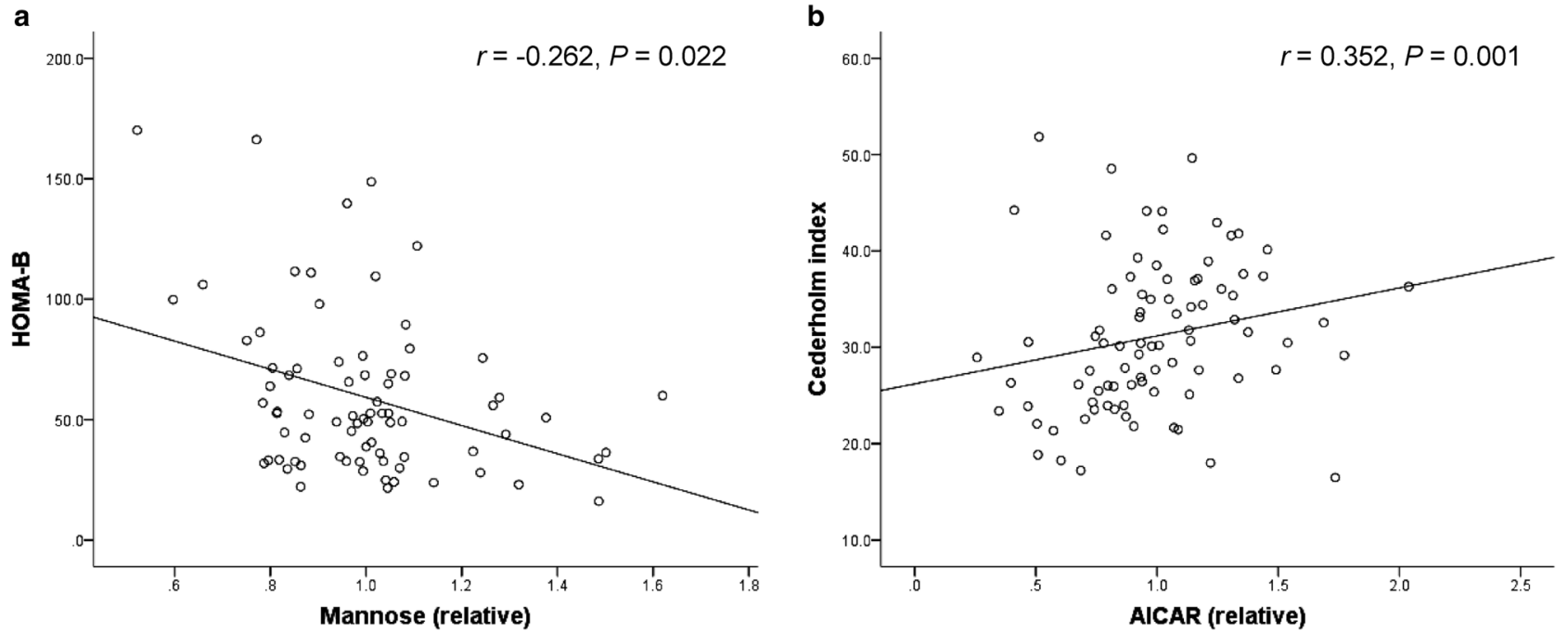

Fig. 3 Correlations of selected metabolites with indices of insulin secretion or insulin sensitivity. (a) Correlation between mannose and HOMA$\mathrm{B}$; (b) correlation between AICAR and the Cederholm index. Data is from the combined set of exploratory set and validation set

regression analysis, we observed that mannose and AICAR were independently associated with HOMA-B $(\beta=-0.316, P=0.001)$ and the Cederholm index $(\beta=0.215, P=0.048)$, respectively, after adjusting for age, $\mathrm{BMI}$, and $\mathrm{HbA}_{1 \mathrm{c}}$ (Supplemental Tables 3 and 4).

Eighty-seven individuals (NC: $n=36 ; \mathrm{FH}: n=65 ; \mathrm{PH}: n=72$ ) were additionally enrolled and assayed with ELISA, the characteristics of which are depicted in Table 3. The mannose levels of $\mathrm{FH}$ $(466.0 \pm 179.3 \mathrm{pg} / \mathrm{ml})$ were numerically higher than those of NC $(409.2 \pm 108.5 \mathrm{pg} / \mathrm{ml})$ and $\mathrm{PH}(390.1 \pm 140.2 \mathrm{pg} / \mathrm{ml})$, respectively, although the differences between the three groups did not reach statistical significance $(P=0.130)$ (Table 3$)$. Regarding AICAR, FH $(523.5 \pm 164.8 \mathrm{pg} / \mathrm{ml})$ and $\mathrm{PH}(512.1 \pm 186.0 \mathrm{pg} / \mathrm{ml})$ had significantly higher AICAR than did NC $(372.8 \pm 90.9 \mathrm{pg} / \mathrm{ml})$ (both $P<$ 0.001 after Bonferroni correction), while $\mathrm{FH}$ and $\mathrm{PH}$ did not differ significantly $(P=0.346)$. Finally, the data from metabolomics and ELISA were transformed to unit SD as (individual value - mean)/ SD and pooled together (NC: $n=36$; FH: $n=65 ; \mathrm{PH}: n=72$ ). The distributions of mannose and AICAR are illustrated in Supplemental Figs. 1A and 1B. Multiple regression analysis revealed that mannose was significantly associated with FPG $(\beta=0.151, P=$ 0.035) after adjusting for age, $\mathrm{BMI}$ and $\mathrm{HbA}_{1 c}$ (Table 4). And mannose was observed to be independently associated with
HOMA-IR $(\beta=0.160, P=0.026)$ but not HOMA-B $(\beta=-0.052, P=$ 0.453 ) (Table 4). We did not observe the significant associations of AICAR with Cederholm index and other parameters $(P>0.05$, data not shown).

\section{DISCUSSION}

Using a non-targeted metabolomics approach, the present study identified 10 differential metabolites between fasting and $\mathrm{PH}$. Of them, mannose was found to be independently associated with FPG and HOMA-IR, suggesting distinct mechanisms of fasting and $\mathrm{PH}$.

In the current study, the analysis of metabolomic data revealed significant link between mannose and HOMA-B, while mannose was found to be independently associated with HOMA-IR in the whole group of participants. In concert with these findings, previous studies have reported that mannose was related with both insulin secretion and insulin sensitivity in humans [13, 14]. Furthermore, an independent association of mannose with FPG was observed, which is consistent with the study by Yoshimura et al. [15] Since the HOMA mode is based on fasting glucose and insulin values, together with the fact that the fasting glucose 
Table 3. The characteristics of the 87 subjects assayed with ELISA

\begin{tabular}{lllll}
\hline Variable & $\mathrm{NC}$ & $\mathrm{FH}$ & $\mathrm{PH}$ & $P$ \\
\hline$n$ (male/female) & $15 / 21$ & $15 / 7$ & $16 / 13$ & 0.139 \\
Age (years) & $49.6 \pm 10.5$ & $50.3 \pm 8.6$ & $51.9 \pm 9.4$ & 0.616 \\
$\mathrm{BMI}\left(\mathrm{kg} / \mathrm{m}^{2}\right)$ & $25.0 \pm 2.6$ & $25.9 \pm 3.6$ & $25.5 \pm 3.3$ & 0.588 \\
$\mathrm{HbA}_{1 \mathrm{c}}(\%)$ & $5.7 \pm 0.4$ & $6.2 \pm 0.5^{*}$ & $6.2 \pm 0.6^{*}$ & $<0.001$ \\
$\mathrm{FPG} \mathrm{(mmol/L)}$ & $5.5 \pm 0.5$ & $7.3 \pm 0.5^{*}$ & $6.0 \pm 0.6^{*+}$ & $<0.001$ \\
2-h PG (mmol/L) & $8.2 \pm 1.8$ & $8.7 \pm 2.1$ & $12.8 \pm 1.3^{*+}$ & $<0.001$ \\
FINS (mU/L) & $9.9 \pm 3.0$ & $12.9 \pm 7.7$ & $10.4 \pm 4.3$ & 0.076 \\
2-h INS (mU/L) & $93.2 \pm 41.9$ & $95.6 \pm 66.8$ & $100.5 \pm 57.3$ & 0.863 \\
HOMA-B & $103.1 \pm 40.0$ & $69.2 \pm 41.3^{*}$ & $89.6 \pm 44.1$ & 0.014 \\
HOMA-IR & $2.4 \pm 0.8$ & $4.2 \pm 2.5^{*}$ & $2.8 \pm 1.2^{\dagger}$ & $<0.001$ \\
Cederholm index & $43.7 \pm 11.3$ & $40.1 \pm 12.2$ & $28.6 \pm 5.0^{*+}$ & $<0.001$ \\
Mannose (pg/ml) & $409.2 \pm 108.5$ & $466.0 \pm 179.3$ & $390.1 \pm 140.2$ & 0.130 \\
AICAR (pg/ml) & $372.8 \pm 90.9$ & $523.5 \pm 164.8^{*}$ & $512.1 \pm 186.0^{*}$ & $<0.001$ \\
\hline
\end{tabular}

$N C$ normal control, $F H$ fasting hyperglycemia, $P H$ post-load hyperglycemia, $B M I$ body mass index, FPG fasting plasma glucose, 2-h PG 2-h plasma glucose, $H b A_{1 c}$ glycated hemoglobin, FINS fasting insulin, 2-h INS 2-h insulin, HOMA-IR the homeostasis model assessment for insulin resistance, HOMA-B the homeostasis model assessment for beta cell function

${ }^{*} P<0.05$ compared with NC after Bonferroni correction

${ }^{+} P<0.05$ compared with FH after Bonferroni correction

Table 4. Multiple regression analysis of the associations of mannose and confounding factors with FPG and HOMA-IR in all subjects

\begin{tabular}{|c|c|c|c|c|}
\hline \multirow{3}{*}{ Independent variable } & \multicolumn{4}{|c|}{ Dependent variable } \\
\hline & \multicolumn{2}{|l|}{ FPG } & \multicolumn{2}{|c|}{ HOMA-IR } \\
\hline & $\beta$ & $P$ & $\beta$ & $P$ \\
\hline Age & -0.023 & 0.763 & 0.031 & 0.684 \\
\hline BMI & -0.119 & 0.099 & 0.425 & 0.000 \\
\hline $\mathrm{HbA}_{1 \mathrm{c}}$ & 0.428 & 0.000 & 0.072 & 0.351 \\
\hline Mannose & 0.151 & 0.035 & 0.160 & 0.026 \\
\hline
\end{tabular}

homeostasis is mainly determined by a feedback loop between the liver and the beta cells [16], it is plausible to speculate that the association of mannose with FPG and HOMA-B/HOMA-IR may, to some extent, reflect the relationship between mannose and hepatic insulin resistance. Mannose is a monosaccharide constituent of glycoproteins and glycolipids. The concentration of free mannose in plasma ranges from 20 to $80 \mu \mathrm{M}$ in healthy adults [17, 18]. It has been shown that the liver is the main site for mannose metabolism [18, 19]. In obese subjects, Lee et al. [13] found significantly lower expression of HK1 and HK2 in the liver, which may lead to decreased utilization of mannose. HK1 and HK2 encode for hexokinase-1 and hexokinase-2, respectively. Hexokinase- 1 and hexokinase- 2 mediate the conversion of mannose to mannose-6-phosphate, which is subsequently involved in the glycoprotein biosynthesis and glycosylation. Abnormal glycosylation has been shown to contribute to insulin resistance in the liver through its impact on insulin receptors [20]. On the other hand, the study in rats by Taguchi et al. [18] reported that hepatic glycogen is a source of plasma mannose. They suggested that stimulated glycogenolysis results in increased mannose 6-phosphate, the hydrolysis of which subsequently leads to increased production of mannose. Oral administration of glucose decreased plasma mannose concentrations in fasted normal rats. However, this effect was blunted in diabetic rats. Intravenous administration of insulin to fed normal rats caused more rapid decrease of mannose than to diabetic rats. In human subjects, Yoshimura et al. [15] observed that the plasma mannose decreased after glucose load in normal controls, but did not decrease in diabetic patients. Collectively, these findings suggest that the decreased utilization (via glycoprotein synthesis) and the increased production (via glycogenolysis) of mannose in the liver contribute to the increase in circulating mannose levels. And mannose may serve as a surrogate marker of hepatic insulin resistance, which merits further investigations.

We observed that, though FINS and $\triangle I_{30} / \triangle G_{30}$ did not differ significantly between $\mathrm{FH}$ and $\mathrm{PH}$. Patients with $\mathrm{PH}$ had higher $2 \mathrm{~h}$ insulin than those with $\mathrm{FH}$, indicating delayed hyper response of insulin in $\mathrm{PH}$. Consistent with this finding, a study using euglycemic-hyperinsulinemic clamp have demonstrated that subjects with isolated impaired glucose tolerance (IGT) had higher late-phase insulin secretion as compared to those with isolated impaired fasting glucose (IFG) [21]. This fact could be partially accounted for by the notion that IGT is featured by extrahepatic insulin resistance while IFG has nearly normal extrahepatic insulin sensitivity $[22,23]$. Patients with IGT need to secrete more insulin to compensate for insulin resistance in the post-absorptive state. Indeed, the insulin sensitivity as measured by Cederholm index was significantly lower in $\mathrm{PH}$ vs. $\mathrm{FH}$ in the current study. Interestingly, we found Cederholm index was significantly associated with AICAR in the metabolomics study. AICAR is an endogenous substance and an intermediate metabolite in the purine de novo synthesis pathway [24]. It activates the AMPsensitive enzymes such as adenosine monophosphate (AMP)activated kinase (AMPK), glycogen phosphorylase, and fructose1,6-bisphosphatase, and thus contributes to oxidative metabolism and mitochondrial biogenesis. It has been reported that treatment with AICAR over an 8-week period can prevent diabetes in Zucker diabetic fatty rats, and insulin sensitivity, as assessed by a hyperinsulinemic-euglycemic clamp, was markedly increased after treatment [25]. Furthermore, AICAR administration was shown to enhance glucose transporter type 4 (GLUT4) protein expression and to increase maximally insulin-stimulated glucose transport in muscles of obese Zucker rats [26]. Additionally, chronic AICARinduced AMPK activation led to significantly reduced visceral and subcutaneous adiposity, increased fat oxidation in white adipocytes, and increased whole-body energy expenditure in Wistar rats. Taken together, these results suggest a link between AICAR and insulin sensitivity.

The present study has several limitations. First, the sample size was relatively small. Although we matched $\mathrm{FH}$ with $\mathrm{PH}$ according to gender, $\mathrm{BMI}$ and $\mathrm{HbA}_{1 \mathrm{c}}$ in order to minimize confounding factors to a great extent, our study may be underpowered for the detection of certain markers specific to $\mathrm{FH}$ or PH. Therefore, this should be regarded as a preliminary study to examine the metabolic features of $\mathrm{FH}$ and $\mathrm{PH}$, and the results should be interpreted with caution. Second, given the cross-sectional nature of the study design, cause and effect relationships could not be established in our study. Finally, instead of using euglycemic insulin clamp, whole-body insulin sensitivity was evaluated with the Cederholm index derived from glucose and insulin concentrations during OGTT. However, it has been demonstrated that OGTTbased indexes correlate well with the results of euglycemic insulin clamp [27], which is the gold standard for measuring insulin sensitivity.

In conclusion, via non-targeted metabolic profiling, we identified that mannose was altered in the state of $\mathrm{PH}$ as compared with $\mathrm{FH}$. Because mannose was shown to be associated with insulin secretion/insulin sensitivity, our findings provide further evidence that distinct physiologic abnormalities characterize $\mathrm{FH}$ and $\mathrm{PH}$. In addition, the results of our study suggest that metabolites could 
be used to stratify different subgroups of diabetes patients and tailor therapeutic interventions accordingly.

\section{ACKNOWLEDGEMENTS}

This work was funded by the National Natural Science Foundation of China (81300666) Natural Science Foundation of Shanghai (17ZR1421300), the Shanghai Municipal Education Commission-Gaofeng Clinical Medicine Grant Support (20161430), and the innovation foundation of translational medicine of Shanghai Jiao Tong University School of Medicine and Shanghai SJTUSM Biobank (15ZH4006). We would like to thank all of the involved clinicians, nurses, and technicians at each of the participating centers for dedicating their time and skill to the completion of this study.

\section{AUTHOR CONTRIBUTIONS}

J.-y.L., J.-h.P. and J.Z. contributed to study design, data analysis, and writing the paper. Y.-n.Z. contributed to data analysis. W.Z., X.-x.H. and L.-W.Y. contributed to conduction of study and data collection. X.-j.M. contributed to study design, data analysis, and interpretation of data. Y.-q.B. and W.-p.J. contributed to interpretation of data and revision of the manuscript.

\section{ADDITIONAL INFORMATION}

Supplementary information is available for this paper at https://doi.org/10.1038/ s41401-018-0018-6.

Competing interests: The authors declare no competing interests.

\section{REFERENCES}

1. Kannel WB, McGee DL. Diabetes and cardiovascular risk factors: the Fra-mingham study. Circulation. 1979;59:8-13.

2. Laakso M. Hyperglycemia and cardiovascular disease in type 2 diabetes. Diabetes. 1999;48:937-42.

3. International Diabetes Federation Guideline Development Group. Guideline for management of postmeal glucose in diabetes. Diabetes Res Clin Pract. 2014;103:256-68.

4. Hanefeld M, Fischer S, Julius U, Schulze J, Schwanebeck U, Schmechel H, et al. Risk factors for myocardial infarction and death in newly detected NIDDM: the Diabetes Intervention Study, 11-year follow-up. Diabetologia. 1996;39:1577-83.

5. Cavalot F, Pagliarino A, Valle M, Di Martino L, Bonomo K, Massucco P, et al. Postprandial blood glucose predicts cardiovascular events and all-cause mortality in type 2 diabetes in a 14-year follow-up: lessons from the San Luigi Gonzaga Diabetes Study. Diabetes Care. 2011;34:2237-43.

6. Coutinho M, Gerstein HC, Wang Y, Yusuf S. The relationship between glucose and incident cardiovascular events. A metaregression analysis of published data from 20 studies of 95,783 individuals followed for 12.4 years. Diabetes Care. 1999:22:233-40.

7. Nicholson JK, Lindon JC, Holmes E. 'Metabonomics': understanding the metabolic responses of living systems to pathophysiological stimuli via multivariate statistical analysis of biological NMR spectroscopic data. Xenobiotica. 1999;29:1181-9.

8. Newgard CB, An J, Bain JR, Muehlbauer MJ, Stevens RD, Lien LF, et al. A branchedchain amino acid-related metabolic signature that differentiates obese and lean humans and contributes to insulin resistance. Cell Metab. 2009;9:311-26.
9. Qiu Y, Cai G, Su M, Chen T, Zheng X, Xu Y, et al. Serum metabolite profiling of human colorectal cancer using GC-TOFMS and UPLC-QTOFMS. J Proteome Res. 2009;8:4844-50.

10. Wang TJ, Larson MG, Vasan RS, Cheng S, Rhee EP, McCabe E, et al. Metabolite profiles and the risk of developing diabetes. Nat Med. 2011;17:448-53.

11. Cederholm J, Wibell L. Insulin release and peripheral sensitivity at the oral glucose tolerance test. Diabetes Res Clin Pract. 1990;10:167-75.

12. Kind T, Wohlgemuth G, Lee DY, Lu Y, Palazoglu M, Shahbaz S, et al. FiehnLib: mass spectral and retention index libraries for metabo-lomics based on quadrupole and time-of-flight gas chromatography/mass spectrometry. Anal Chem. 2009;81:10038-48.

13. Lee $S$, Zhang C, Kilicarslan M, Piening BD, Bjornson E, Hallstrom BM, et al. Integrated network analysis reveals an association between plasma mannose levels and insulin resistance. Cell Metab. 2016;24:172-84.

14. Mardinoglu A, Stancakova A, Lotta LA, Kuusisto J, Boren J, Bluher M, et al. Plasma mannose levels are associated with incident type 2 diabetes and cardiovascular disease. Cell Metab. 2017;26:281-3.

15. Yoshimura K, Hirano S, Takata H, Funakoshi S, Ohmi S, Amano E, et al. Plasma mannose level, a putative indicator ofglycogenolysis, and glucose tolerance in Japanese individuals. J Diabetes Investig. 2017;8:489-95.

16. Turner RC, Holman RR. Pathophysiology of diabetes. Lancet. 1976;2:856.

17. Sone $H$, Shimano $H$, Ebinuma $H$, Takahashi A, Yano $Y$, lida KT, et al. Physiological changes in circulating mannose levels in normal, glucose-intolerant, and diabetic subjects. Metabolism. 2003;52:1019-27.

18. Taguchi T, Miwa I, Mizutani T, Nakajima H, Fukumura Y, Kobayashi I, et al. Determination of D-mannose in plasma by HPLC. Clin Chem. 2003;49:181-3.

19. Davis JA, Freeze HH. Studies of mannose metabolism and effects of long-term mannose ingestion in the mouse. Biochim Biophys Acta. 2001;1528:116-26.

20. Caro JF, Cecchin F, Sinha MK. Is glycosylation in the liver needed for insulin binding, processing, and action? Evidence for heterogeneity. J Biol Chem. 1984;259:12810-6.

21. Abdul-Ghani MA, Jenkinson CP, Richardson DK, Tripathy D, DeFronzo RA. Insulin secretion and action in subjects with impaired fasting glucose and impaired glucose tolerance: results from the Veterans Administration Genetic Epidemiology Study. Diabetes. 2006;55:1430-5.

22. Abdul-Ghani MA, Tripathy D, DeFronzo RA. Contributions of beta-cell dysfunction and insulin resistance to the pathogenesis of impaired glucose tolerance and impaired fasting glucose. Diabetes Care. 2006;29:1130-9.

23. Basu R, Barosa C, Jones J, Dube S, Carter R, Basu A, et al. Pathogenesis of prediabetes: role of the liver in isolated fasting hyperglycemia and combined fasting and postprandial hyperglycemia. J Clin Endocrinol Metab. 2013;98: E409-17.

24. Sabina RL, Holmes EW, Becker MA. The enzymatic synthesis of 5-amino-4-imidazolecarboxamide riboside triphosphate (ZTP). Science. 1984;223:1193-5.

25. Pold R, Jensen LS, Jessen N, Buhl ES, Schmitz O, Flyvbjerg A, et al. Long-term AICAR administration and exercise prevents diabetes in ZDF rats. Diabetes. 2005;54:928-34.

26. Buhl ES, Jessen N, Pold R, Ledet T, Flyvbjerg A, Pedersen SB, et al. Long-term AICAR administration reduces metabolic disturbances and lowers blood pressure in rats displaying features of the insulin resistance syndrome. Diabetes. 2002;51:2199-206.

27. Matsuda M, DeFronzo RA. Insulin sensitivity indices obtained from oral glucose tolerance testing: comparison with the euglycemic insulin clamp. Diabetes Care. 1999;22:1462-70. 